\title{
Ultrafast manipulation of hard $x$-rays by efficient Bragg switches
}

\author{
M. Herzog, ${ }^{1}$ W. Leitenberger, ${ }^{1}$ R. Shayduk, ${ }^{2}$ R. M. van der Veen, ${ }^{3,4}$ C. J. Milne, ${ }^{3,4}$ \\ S. L. Johnson, ${ }^{3}$ I. Vrejoiu, ${ }^{5}$ M. Alexe ${ }^{5}$ D. Hesse, ${ }^{5}$ and M. Bargheer ${ }^{1,2,6, a)}$ \\ ${ }^{1}$ Institut für Physik und Astronomie, Universität Potsdam, Karl-Liebknecht-Str. 24-25, \\ 14476 Potsdam, Germany \\ ${ }^{2}$ Helmholtz-Zentrum Berlin für Materialien und Energie GmbH, Hahn-Meitner-Platz, 1, \\ 14109 Berlin, Germany \\ ${ }^{3}$ Swiss Light Source, Paul Scherrer Institut, 5232 Villigen PSI, Switzerland \\ ${ }^{4}$ Laboratoire de Spectroscopie Ultrarapide, Ecole Polytechnique Fédéral de Lausanne, 1015 Lausanne, \\ Switzerland \\ ${ }_{5}^{5}$ Max-Planck-Institut für Mikrostrukturphysik, Weinberg 2, 06120 Halle, Germany \\ ${ }^{6}$ Max-Planck-Institut für Kolloid- und Grenzflächenforschung, Am Mühlenberg 1, 14476 Potsdam-Golm, \\ Germany
}

(Received 24 February 2010; accepted 30 March 2010; published online 23 April 2010)

\begin{abstract}
We experimentally demonstrate efficient switching of the hard x-ray Bragg reflectivity of a $\mathrm{SrRuO}_{3} / \mathrm{SrTiO}_{3}$ superlattice by optical excitation of large-amplitude coherent acoustic superlattice phonons. The rocking curve changes drastically on a 1 ps timescale. The $\left(\begin{array}{lll}0 & 0 & 116\end{array}\right)$ reflection is almost extinguished $\left(\Delta R / R_{0}=-0.91\right)$, while the $\left(\begin{array}{lll}0 & 0 & 118\end{array}\right)$ reflection increases by more than an order of magnitude $\left(\Delta R / R_{0}=24.1\right)$. The change of the $\mathrm{x}$-ray structure factor depends nonlinearly on the phonon amplitude, allowing manipulation of the $\mathrm{x}$-ray response on a timescale considerably shorter than the phonon period. Numerical simulations for a superlattice with slightly changed geometry and realistic parameters predict a switching-contrast ratio $\Delta R / R_{0}$ of 700 with high reflectivity. (C) 2010 American Institute of Physics. [doi:10.1063/1.3402773]
\end{abstract}

Ultrafast x-ray techniques have become more and more prevalent in various scientific disciplines that are interested in directly observing atomic motion in real time. Very recently the first hard $\mathrm{x}$-ray free electron laser has started operation, ${ }^{1}$ and similar large-scale facilities are being constructed. In the meantime, widely available synchrotron radiation sources are usually limited to $\sim 100$ ps pulses. Faster time resolution at these sources has been realized in a few cases by ultrafast modulation of the electron beam in the storage ring (slicing or low-alpha mode), ${ }^{2-5}$ or by using ultrafast streak camera detectors to achieve time resolutions down to several hundred femtoseconds. ${ }^{6-8}$ In such cameras a photocathode converts the x-ray pulse into an electron pulse with similar time structure, and this electron pulse is subsequently manipulated with rapidly varying electric fields. So far, an efficient device to manipulate the time structure of a hard x-ray pulse itself is missing, although some concepts using coherent optical phonons in crystals have been discussed theoretically. 9,10

Here, we report on ultrafast $\mathrm{x}$-ray diffraction measurements that demonstrate vast changes of the various Bragg peaks of a $\mathrm{SrRuO}_{3} / \mathrm{SrTiO}_{3}$ (SRO/STO) superlattice on a timescale of 1 ps. These modulations are triggered by the excitation of the structure with femtosecond laser pulses. As a consequence, the $\left(\begin{array}{lll}0 & 0 & 116\end{array}\right)$ superlattice reflection is strongly reduced, whereas the $\left(\begin{array}{lll}0 & 0 & 118\end{array}\right)$ reflection simultaneously exhibits a huge increase. The results are compared to numerical simulations evidencing that the change of the $\mathrm{x}$-ray structure factor for the $\left(\begin{array}{lll}0 & 0 & 118\end{array}\right)$ superlattice peak depends nonlinearly on the phonon amplitude. This results in a nonsinusoidal modulation of the peak intensity in time. The

\footnotetext{
a) Author to whom correspondence should be addressed. Electronic mail: bargheer@uni-potsdam.de.
}

$\mathrm{x}$-ray response can thus be manipulated on a timescale shorter than the phonon period, leading to ultrafast $\mathrm{x}$-ray pulses with a full width at half maximum of $\Delta T=1 \mathrm{ps}$. Finally, we show numerical simulations of the rocking curve of a superlattice with realistic parameters predicting a switching-contrast ratio $\Delta R / R_{0}$ of 700 for such ultrafast gating of $\mathrm{x}$-rays. This demonstrates the potential application of superlattices as an ultrafast Bragg switch.

The investigated superlattice consists of ten bilayers of the perovskite oxides SRO and STO terminated by a STO capping layer. The thin layers were epitaxially grown by pulsed laser deposition along the [001] direction on top of a STO substrate with a $42 \mathrm{~nm}$ thick SRO buffer layer. ${ }^{11,12}$ For sample characterization static $\mathrm{x}$-ray diffraction measurements [Fig. 1(a)] with a high x-ray flux at $8818 \mathrm{eV}$ have been performed at the EDR beamline of BESSY II. ${ }^{13}$ The ultrafast $\mathrm{X}$-ray experiments were carried out at the FEMTO-slicing beamline of the Swiss Light Source (SLS) (Ref. 4) with $\mathrm{x}$-ray pulses of $140 \pm 30$ fs pulse duration, a photon energy of $5200 \mathrm{eV}$ and a mean photon number of $\sim 20$ photons per $\mathrm{x}$-ray pulse. ${ }^{14} \mathrm{~A}$ femtosecond Ti:Sa laser system generating the $800 \mathrm{~nm}$ excitation pulses with $115 \mathrm{fs}$ pulse duration was synchronized to the $\mathrm{x}$-ray pulses at a repetition rate of $1 \mathrm{kHz}$. The relatively low $\mathrm{x}$-ray flux consequently results in a smaller signal-to-noise ratio as seen in Fig. 1(b), which shows the measured diffracted x-ray intensity $I_{d}$ normalized by the incident x-ray intensity $I_{i}$. We call $R=I_{d} / I_{i}$ the Bragg reflectivity, or short, reflectivity. In addition, the data sets were corrected for the so called halo background, which is generated by incompletely damped electrons from previously sliced bunches. ${ }^{4}$

The high structural perfection of the sample is evident from the very sharp peaks in the static rocking curve in Fig. 1(a). In order to deduce the structural parameters of the su- 


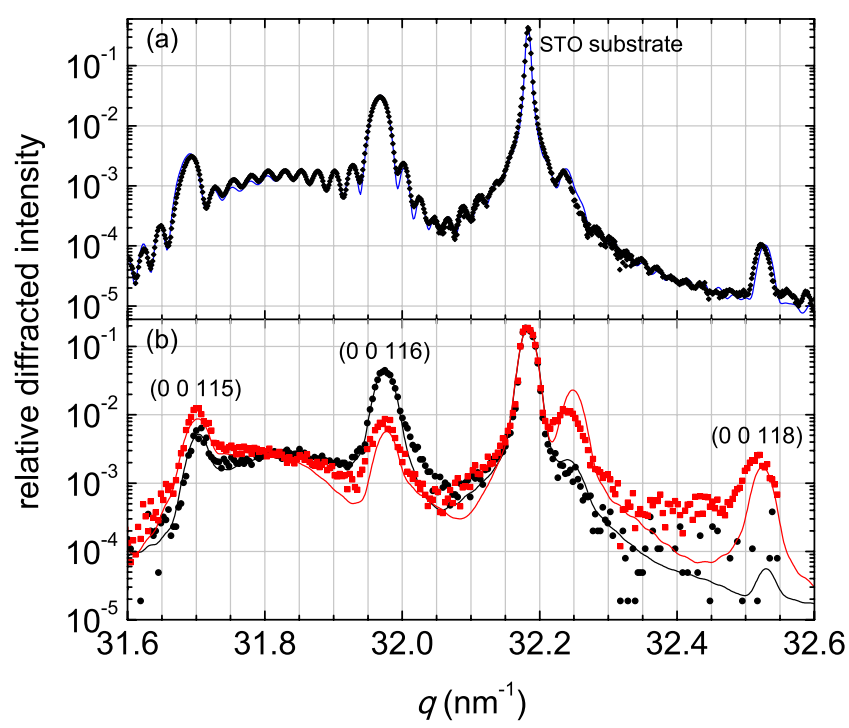

FIG. 1. (Color online) (a) Black diamonds: static rocking curve recorded at BESSY II with full x-ray flux. (b) Rocking curve of the unexcited SRO/STO superlattice (black bullets) and $1.6 \mathrm{ps}$ after laser pulse excitation with a fluence of $37 \mathrm{~mJ} / \mathrm{cm}^{2}$ at $800 \mathrm{~nm}$ (red squares). These data sets were measured at the SLS in FEMTO-slicing mode. The solid lines are the corresponding numerical simulations.

perlattice, numerical simulations based on dynamical diffraction theory have been performed, which also account for the Debye-Waller effect and interface roughness. The simulation [solid line in Fig. 1(a)] yields the following parameters for the individual layers: Layer thickness $d_{\mathrm{SRO}}=5.54 \mathrm{~nm}$ and $d_{\mathrm{STO}}=12.94 \mathrm{~nm}$ and out-of-plane lattice constant of the perovskite unit cell $c_{\mathrm{SRO}}=0.3957 \mathrm{~nm}$ and $c_{\mathrm{STO}}=0.3921 \mathrm{~nm}$. Moreover, interface layers of intermixed SRO and STO unit cells with $d_{\text {mix }}=1.96 \mathrm{~nm}$ and $c_{\text {mix }}=0.3923 \mathrm{~nm}$ had to be assumed to simulate the data. ${ }^{15}$

When the sample is excited by an optical pulse, the rocking curve varies drastically on an ultrafast timescale. The largest intensity changes are observed when the x-ray pulse probes the transient structure at $t=1.6 \mathrm{ps}$ after the pump pulse. The corresponding rocking curve for a pump fluence of $37 \mathrm{~mJ} / \mathrm{cm}^{2}$ is shown in Fig. 1(b) (red squares). The diffracted x-ray intensity exhibits a relative increase of $\Delta R^{118} / R_{0}^{118}=13.7$ for the $\left(\begin{array}{lll}0 & 0 & 118\end{array}\right)$ superlattice reflection and a respective decrease of $\Delta R^{116} / R_{0}^{116}=-0.80$ for the ( $\left.\begin{array}{lll}0 & 0 & 116\end{array}\right)$ reflection.

In Fig. 2, the temporal evolution of these two Bragg peak intensities is plotted for a higher excitation fluence of $53 \mathrm{~mJ} / \mathrm{cm}^{2} . R^{116}(t)$ shows an oscillatory response that resembles a damped cosine function with a period of $3.2 \mathrm{ps}$. The diffraction efficiency of the $(00116)$ reflection is maximally suppressed at $t=1.6 \mathrm{ps}$ down to $0.09 \times R_{0}^{116}$. The transient Bragg reflectivity $R^{118}(t)$ exhibits the same period but clearly shows peaks having a full width at half maximum of $\Delta T=1$ ps rather than a sinusoid. Thus, widely available $\sim 100$ ps x-ray pulses from synchrotron radiation sources reflected from the sample at $\left(\begin{array}{lll}0 & 0 & 118\end{array}\right)$ would be shaped into $1 \mathrm{ps}$ bursts as opposed to a sine wave. The maximum relative change of the diffracted intensity of the $(00118)$ reflection is $\Delta R^{118} / R_{0}^{118}=24.1$.

The physical mechanism behind this transient $\mathrm{x}$-ray response can be understood as follows. The optical pump light is absorbed in the metallic SRO layers exclusively, leading to

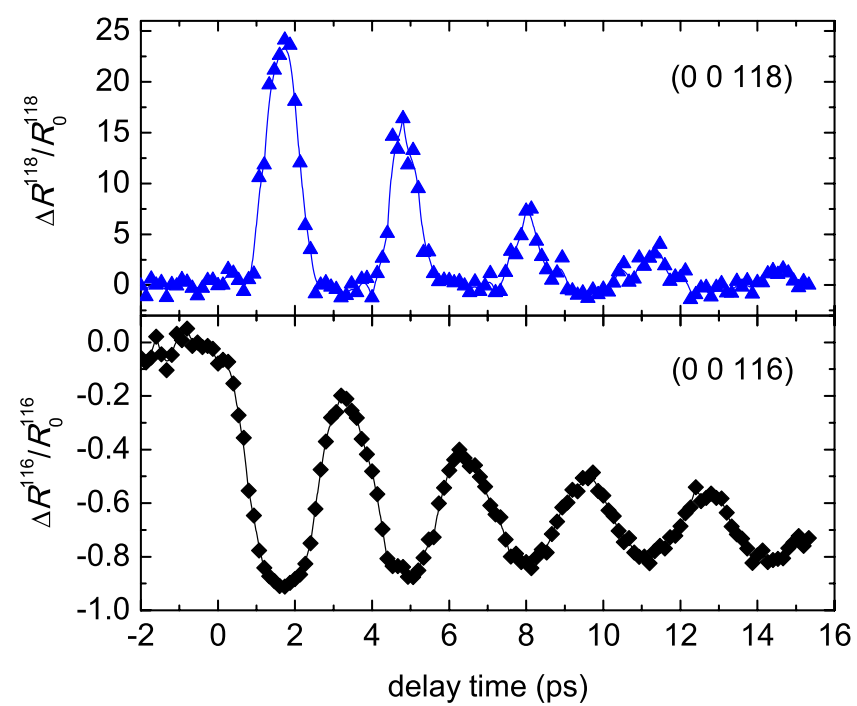

FIG. 2. (Color online) Relative change of the transient x-ray Bragg reflectivity of superlattice reflection (l $\left.\begin{array}{lll}0 & 0 & 116\end{array}\right)$ (black diamonds) and $\left(\begin{array}{lll}0 & 0 & 118\end{array}\right)$ (blue triangles) after excitation $\left(\lambda=800 \mathrm{~nm}\right.$ and fluence $\left.53 \mathrm{~mJ} / \mathrm{cm}^{2}\right)$. The solid lines are guidelines to the eye.

an expansion of these layers. At short timescales this expansion must be compensated by a compression of the optically transparent STO layers, as they are squeezed equally from both sides. Essentially, the induced atomic motion corresponds to a standing longitudinal acoustic wave with a wavelength determined by the spatial double-layer period $d_{\mathrm{SL}}$ of the superlattice. The temporal period of this coherent acoustic phonon mode corresponds to the time $T_{\mathrm{ph}}=d_{\mathrm{SL}} / v_{\mathrm{av}}$ it takes a sound wave to propagate through one SRO/ STO double layer with thickness $d_{\mathrm{SL}}=d_{\mathrm{SRO}}+d_{\mathrm{STO}}+2 d_{\text {mix }}$ $=22.8 \mathrm{~nm}$ at the average longitudinal sound velocity $v_{\mathrm{av}} \approx 7.3 \mathrm{~nm} / \mathrm{ps} \quad\left[v_{\mathrm{SRO}}=6.3 \mathrm{~nm} / \mathrm{ps}\right.$ (Ref. 16) and $v_{\mathrm{STO}}$ $=7.8 \mathrm{~nm} / \mathrm{ps}$ (Ref. 17)]. This explains the measured phonon period of $3.2 \mathrm{ps}$.

For simplicity, we assume homogeneous strain in identical layers for the numerical simulations of the rocking curves of the optically excited structure. The solid lines in Fig. 1(b) correspond to zero strain (black solid line) and $1.6 \%$ strain (red solid line) in SRO, respectively. For the larger fluence (Fig. 2) we calculate a SRO peak strain of $1.8 \%$. The very good agreement between the calculated curves and the experimental data already indicates that the simulations correctly account for the induced transient structural changes, i.e., an expansion of SRO and a compression of STO (the intermixed interface layers are kept constant). The model has been previously applied for different samples as well. ${ }^{18}$

In order to emphasize the potential of superlattices as a means for manipulating x-rays on ultrafast timescales, we have performed numerical simulations for structures with only slightly different geometries which, however, exhibit greatly improved switching contrasts. Essentially, the layer thicknesses have been varied in order to maximally suppress the $\left(\begin{array}{lll}0 & 0 & 118\end{array}\right)$ reflection in the unexcited state. In the experiments reported above the dynamics were completely reversible, that is, the samples were not damaged. We therefore applied the identical SRO strain level in the simulations for the theoretical structure. Figure 3 shows the simulated rocking curves for zero strain (dashed line) and 1.8\% peak strain (solid line) in the SRO layers of a superlattice with the thick- 


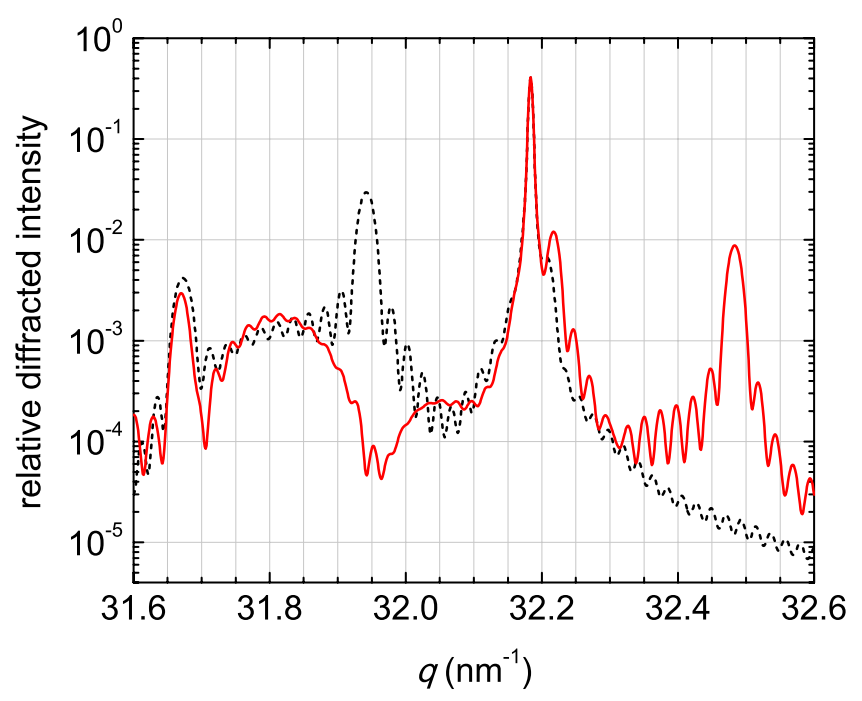

FIG. 3. (Color online) Simulation of tailored SRO/STO superlattice with a maximized contrast ratio of the $\left(\begin{array}{llll}0 & 0 & 118\end{array}\right)$ reflection. The black dashed (red solid) line shows the rocking curve of the stationary (excited) superlattice.

ness parameters $d_{\mathrm{SRO}}=8.7 \mathrm{~nm}$ and $d_{\mathrm{STO}}=14.5 \mathrm{~nm}$, and the same out-of-plane lattice constants as stated above. ${ }^{19}$ The intensity of the $\left(\begin{array}{lll}0 & 0 & 118\end{array}\right)$ reflection changes by a factor of $\Delta R / R_{0}=700$ up to a reflectivity of $1 \%$ of the incident x-ray intensity. However, the nonlinear response to the strain still holds and therefore the corresponding time structure after laser excitation is basically identical to the behavior shown in Fig. 2. The switching contrast could be improved even further by changing the constituent materials in the layers.

In conclusion, we have demonstrated that the optical excitation of superlattice structures by femtosecond laser pulses can be exploited to manipulate hard $\mathrm{x}$-rays on a 1 ps timescale. In contrast to previous suggestions, the crucial idea of this concept is to modulate the diffraction efficiency of stationary forbidden superlattice Bragg peaks with very low background by laser excitation of large-amplitude high-wave vector acoustic phonons. We have experimentally shown both switching the x-ray reflectivity on and off by using different superlattice diffraction orders of the very same SRO/ STO superlattice. Numerical simulations with realistic structural parameters suggest that the performance can be greatly improved by further tailoring the epitaxial structures. The presented experimental method may be used to generate $1 \mathrm{ps}$ $\mathrm{x}$-ray pulses from conventional $\sim 100$ ps pulses in electron storage rings for ultrafast $\mathrm{x}$-ray diffraction experiments.

The time-resolved experiments were performed on the X05LA beamline at the Swiss Light Source, Paul Scherrer Institut, Villigen PSI, Switzerland. We thank the DFG for supporting the project via Grant Nos. BA 2281/3-1 and SFB 762.

${ }^{1}$ B. McNeil, Nat. Photonics 3, 375 (2009).

${ }^{2}$ R. Schoenlein, S. Chattopadhyay, H. Chong, T. Glover, P. Heimann, C. Shank, A. Zholents, and M. Zolotorev, Science 287, 2237 (2000).

${ }^{3}$ S. Khan, K. Holldack, T. Kachel, R. Mitzner, and T. Quast, Phys. Rev. Lett. 97, 074801 (2006).

${ }^{4}$ P. Beaud, S. Johnson, A. Streun, R. Abela, D. Abramsohn, D. Grolimund, F. Krasniqi, T. Schmidt, V. Schlott, and G. Ingold, Phys. Rev. Lett. 99, 174801 (2007).

${ }^{5}$ M. Abo-Bakr, J. Feikes, K. Holldack, P. Kuske, W. B. Peatman, U. Schade, G. Wüstefeld, and H.-W. Hübers, Phys. Rev. Lett. 90, 094801 (2003).

${ }^{6}$ Z. Chang, A. Rundquist, J. Zhou, M. M. Murnane, H. C. Kapteyn, X. Liu, B. Shan, J. Liu, L. Niu, M. Gong, and X. Zhang, Appl. Phys. Lett. 69, 133 (1996).

${ }^{7}$ J. Liu, J. Wanga, B. Shan, C. Wang, and Z. Chang, Appl. Phys. Lett. 82, 3553 (2003).

${ }^{8}$ J. Feng, H. J. Shin, J. R. Nasiatka, W. Wan, A. T. Young, G. Huang, A. Comin, J. Byrd, and H. A. Padmore, Appl. Phys. Lett. 91, 134102 (2007)

${ }^{9}$ P. Bucksbaum and R. Merlin, Solid State Commun. 111, 535 (1999).

${ }^{10}$ J. Sheppard, P. Sondhauss, R. Merlin, P. Bucksbaum, R. Leed, and J. Wark, Solid State Commun. 136, 181 (2005).

${ }^{11}$ I. Vrejoiu, G. L. Rhun, L. Pintilie, D. Hesse, M. Alexe, and U. Gösele, Adv. Mater. (Weinheim, Ger.) 18, 1657 (2006).

${ }^{12}$ C. v. Korff Schmising, A. Harpoeth, N. Zhavoronkov, Z. Ansari, C. AkuLeh, M. Woerner, T. Elsaesser, M. Bargheer, M. Schmidbauer, I. Vrejoiu, D. Hesse, and M. Alexe, Phys. Rev. B 78, 060404(R) (2008).

${ }^{13}$ Energy bandwidth $2 \times 10^{-4}$, divergence $270 \mu \mathrm{rad}, p$-polarization.

${ }^{14}$ Energy bandwidth $1.5 \times 10^{-4}$, vertical convergence of $840 \mu \mathrm{rad}$ due to focussing, $s$-polarization.

${ }^{15}$ The parameters for the simulations of the SLS data had to be slightly adjusted within the structural inhomogeneities.

${ }^{16}$ S. Yamanaka, T. Maekawa, H. Muta, T. Matsuda, S. Kobayashi, and K. Kurosaki, J. Solid State Chem. 177, 3484 (2004).

${ }^{17}$ Y. H. Ren, M. Trigo, R. Merlin, V. Adyam, and Q. Li, Appl. Phys. Lett. 90, 251918 (2007).

${ }^{18}$ C. v. Korff Schmising, M. Bargheer, M. Kiel, N. Zhavoronkov, M. Woerner, T. Elsaesser, I. Vrejoiu, D. Hesse, and M. Alexe, Phys. Rev. Lett. 98, 257601 (2007).

${ }^{19}$ The assumption of perfect interfaces in the simulation, i.e., no intermixing, is not crucial. The presence of three intermixed unit cells would diminish the switching ratio by less than a factor of 2 with almost unchanged maximum reflectivity. 